\title{
Design and Research on Split Timing System of Horse Racing
}

\author{
Hanhua Zhang \\ International School of Equestrian \\ Wuhan Business University \\ Wuhan, China 430056
}

\author{
Shuang Zhang* \\ International School of Equestrian \\ Wuhan Business University \\ Wuhan, China 430056 \\ *Corresponding Author
}

\begin{abstract}
This paper studies the current status of the split timing system of horse racing at home and abroad through literature review, expert interviews and field survey. According to the results, the current split time devices are used for manual operation. And they can only determine the subtime of the first horse. The manual device can't meet the requirements of the horse race in accuracy and operating speed. In view of this, this study designed an automatic split timing system. We can quickly and accurately record the race time of each horse in the race. And this record would provide valid reference data for horse racing, provide intensity index to train the horse for the trainer, provide scientific basis for the decision-making of caballero, and provide technical support for the development of the horse racing industry.
\end{abstract}

Keywords—horse race; split time; design

\section{INTRODUCTION}

The split timing system of horse racing is to measure the time used by the race horse over the specified distance. It is the device. The device is professional and rare. According to the survey, the device is not available on the market. Due to the particularity of the equipment, if the equipment is needed at domestic and international racecourses, the racecourse can be customized with professional companies according to the needs of its own enterprises. The customized equipment produced by such cooperation is very expensive. At present, most of the racecourses use the software of manual operation to split time. This manual operation software does not involve too much external hardware. It is only the cost of software development. And the price is more reasonable for most of the racehorses. However, such manual operation software is cumbersome in operation. And it has low accuracy. Also, it is greatly affected by the reaction speed and the movement speed of the operator. In the contemporary era of information popularization, we need to solve the problem of split timing in horse racing to adapt to the rapid development of China's horse racing industry from the perspective of information. To this end, this study specially designed an automated split timing system to simplify the operation flow, improve measurement accuracy and speed, and provide high-quality technical service support for the development of horse racing industry.

\section{THE INTRODUCTION OF MANUAL SPLIT TIMING SYSTEM}

\section{A. The Basic Principles of Manual Split Timing System}

Manual split timing system is implemented by software, and the software needs to run on a normal computer. The operation of the system requires the cooperation of two staff members. One operator is responsible for operating the computer, and the other operator (it is also could be an observer) needs to observe the real-time position of the first horse in the race through the telescope. Computer operators can only focus on the operation of split timing software. He has no time to observe race conditions of horses in the arena. The observers should observe the real-time position of the horses in real time through telescopes. He also has no time to make computer operations. In order to achieve the split timing, the operator and the observer need to have communication timely and accurately. The mistakes of each side will result in inaccurate recording time. At the beginning of the race, the computer operator gets the command to start the race through the walkie-talkie and quickly launches the split timing system of the software. During the competition, the observer should observe whether the horse passes the distance signboard set on the inner guardrail through the telescope. If the head of the first horse touches the distance signboard, the operator should inform the computer operator through the voice communication. And the computer operator will quickly press the time button to record the time when the horse passes the first signboard. In a similar way, the record of the remaining split time is taken in the same way until the contest is over. The main difficulty of the manual split timing system is the judgment on the horse's position in the race, the speed and accuracy of the operator, communication speed and operating speed.

\section{B. The Defects of Manual Split Timing System}

Manual split timing system has the following disadvantages. First, it needs many operators. The entire race requires two staff to work together, which will lead to the increase of the costs of entire race. The second is the lack of precision and speed. The operation of manual split timekeeping system belongs to manual operation. The accuracy of time is greatly influenced by the computer 
operator's reaction speed and movement speed, and also influenced by the observer's reaction speed. The maximum speed of horse race can exceed $70 \mathrm{~km} / \mathrm{h}$. And there are many horses in the race. It is difficult for the observer to quickly respond to the position information of the horses. Third, the error is large. The observer would stand at a fixed position to observe the real-time position of the horse in the arena. The track is oval. There would have the visual error in observing the different signboard through the fixed points. And the observed results are inconsistent with the actual results. Then, it would have large errors. Fourth, we can't measure the split time of all the participating horses in the competition. Due to the slow response of the human beings, the horses run fast. And the number of horses participating in the competition is large. In the contest, the ranking changes frequently. Therefore, it is hard to record the split time manually for all partic ipating horses. In the official races, the split time of the horses recorded by the manual split-time systemis the first horse in the race in real time.

\section{The Design OF AUt OMATIC Split Timing System}

\section{A. The Design Principles of Automatic Split Timing System}

Automatic split timing system uses information technology to solve the split time of each horse in the horse racing. The system requires putting an electronic chip in the noseband of each horse, which is used to launch the horse's real-time position information at a range of 50 meters. The receiving device of position information is arranged at distance signboard. And it could receive vertically the position information transmitted by all the electronic chips in the horse noseband. Also, it could transmit the position information to the general service station to count the split time through the wireless methods. The system service station starts the timing device through the wireless signal at the start of the race, receives the horse's position information during the horse race, combines the position information with the time information to calculate each horse's split time and send it to the electronic display.

\section{B. Function Module of Automatic Split Timing System}

1) Starting signal ejector: Starting signal ejector is a wireless device. And the main role is to receive the start signal, and send the signal to the total service station. The device is connected with the opening device of the brake box. And it can start the signal ejector while the brake box is opened. Then, it would realize the synchronization of the brake box opening and the timing opening. The transmission distance of starting signal ejector should not be less than $3 \mathrm{~km}$. The device model is shown in "Fig. 1".

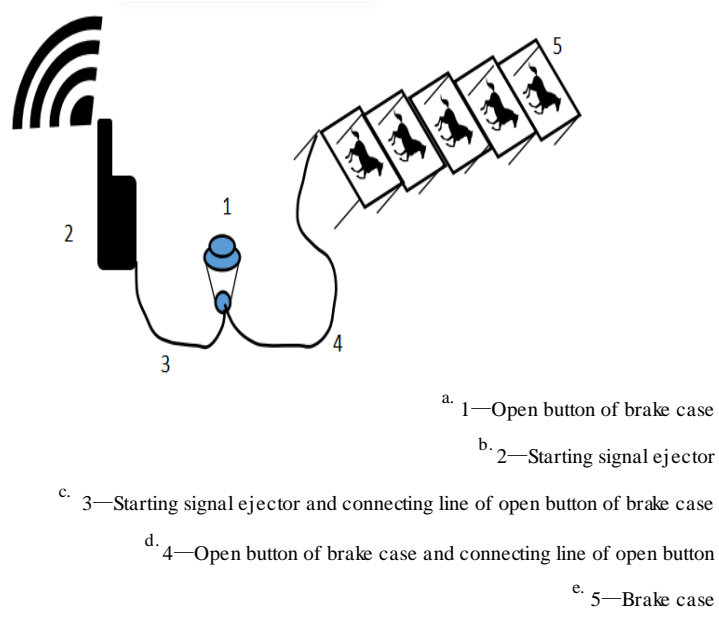

Fig. 1. Starting signal eject or and brake box.

2) Location information receiving and transmitting device: The location information receiving and transmitting device is used to receive the location information of the horse passing the distance signboard. And it would send the location information to the main service station. The device is able to vertically sense the exact position of the positioning chip in the nose band when the horses pass the distance signboard. The most important problem of the device is the accuracy. The higher the accuracy is, the more accurate the determination of the split time is. The device model is shown in "Fig. 2".

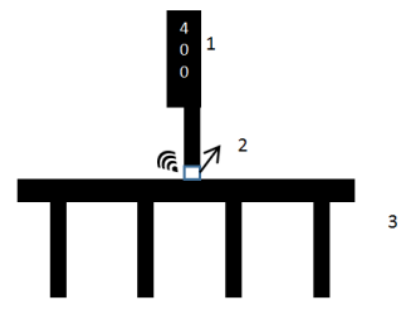

f. 1 - distance signboard

g. 2 -location information receiving and transmitting device

h. 3 -inner fence

Fig. 2. Location information receiving and transmitt ing device connection of dist ance signboard.

3) Noseband and positioning chip: The noseband is a device worn on the nose of a horse. The noseband is specially designed with a void in the middle for positioning the chip. Positioning chip mounting and unloading must be convenient. In the process of running, it has a strong stability. And it can't fall off. The purpose of the positioning chip is to send the location information of the chip to the location information receiving and transmitting device, and there is no interference between the positioning chips. The noseband and positioning chip are shown in "Fig. 3". 


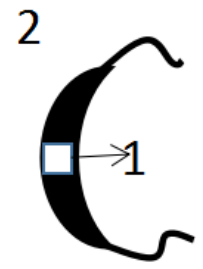

Fig. 3. The noseband and positioning chip.

4) Information receiving device: The information receiving device is used to receive the launching information and the position information transmitted by the "starting signal ejector" and the "position information receiving and transmitting device". It sent starting information and location information through the usb connection to the total service station. Information receiving device is shown in "Fig. 4".

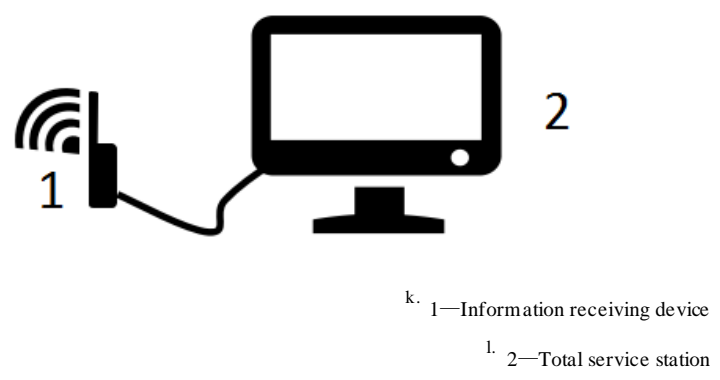

Fig. 4. Information receiving device.

5) Total service station: The total service station is used for the processing storage, query and display of all information. The total service station includes the following functional modules: display module of split time statistics of all the horses; display module of the split time of first horse; information query processing module of the past data. The display module of the split time of all the horses is used to display the time when the horse passes the signboard. And we can view the information of single horse's data according to the needs. The display module of the split time of the first horse is used to statistically show all the split time of the first horse in the race. Due to the variability of horse rankings in race, it should process the time. For example, in the 400 meters, the second horse may surpass the first horse. And in the 800 meters, the No. 5 horse may be the leader. Therefore, the main task of the function module is to determine the split time of the horses passing the signboard. And it can't display the split time of single horse. The query processing module of historical data information is used for querying the time information of all the horses in all the events. And the information is of great significance to the analysis of the athletic performance of the horses. The mode pattern of total service station is shown in "Fig. 5".

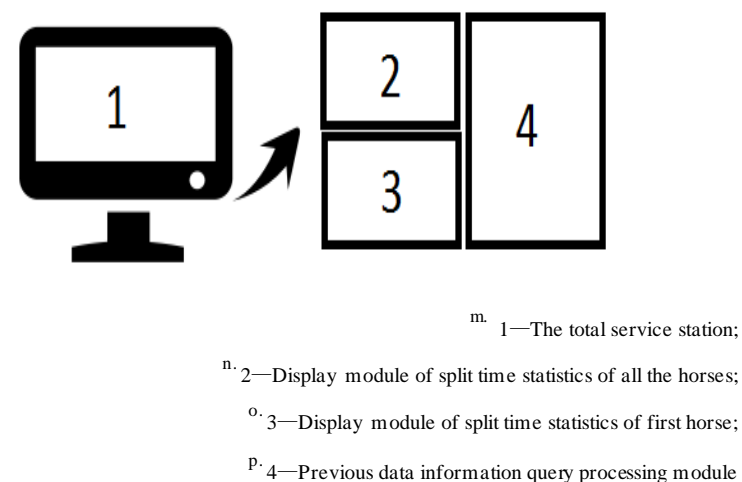

Fig. 5. Total servicestation.

6) Ideograph of working principle: The coordination working principle of auto matic split timing system is shown in "Fig. 6".

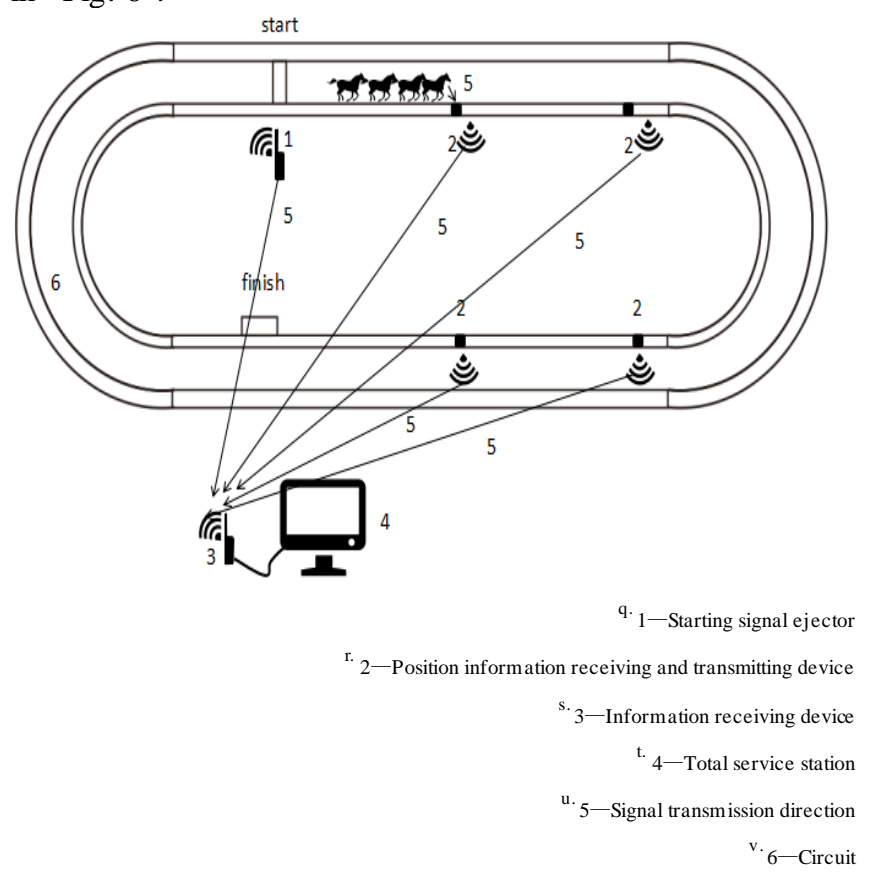

Fig. 6. The coordination working principle of automatic split timing system.

\section{THE ADVANT AGES AND APPLICATIONS OF AUTOMATIC SPLIT TIMING SYSTEM}

\section{A. Advantages of Automatic Split Timing System}

The advantage of automatic split timing system is organically to connect the start-up with the timing closely. And it would achieve the automatic processing of split timing. Also, it reduces the workload and improves the accuracy and speed. At the same time, the automatic split timing system has the function of data storage, which can store the split time of all the participating horses in the database. And it can retrieve the data information at any time according to the need, which provides the data support for the analysis of athletic ability of the horses. 


\section{B. The Application of Automatic Split Timing System}

The time recorded by the split timing system is mainly used in the analysis of the ability of the horse, the horse racing, and the daily training of horses and the walk of the jockey. First, it is to analyze the athletic ability of horses. Through the split timing, we can understand the athletic characteristics of the horses and distinguish the type of horse running. Second, it is horse racing. The fans can know the time spent by the horse at each distance through the split timing. And the horse fans can make the betting according to the time information. Third, it is the daily training of horses. According to the information of the split time, we could adjust the training. If the split time of horses in the training is too long, the training of horses is not enough. Fourth: it is the walk position of jockey in the area. According to the different split time of the horses, the jockey can determine how to ride a horse in the race, and the acceleration and deceleration in different sectors, etc. This can provide a reference for the jockey to make decisions during the race.

\section{CONCLUSION}

- There are many disadvantages of manual split timing. It needs many operators, lacks the accuracy and speed, and can't measure all the split time of participating horses in the race. And manual split timing equipment is not suitable for the race.

- Automatic split timing system has many advantages such as automated processing, low workload, high accuracy and speed and so on. And this is the development trend in the future.

- Split timing system is very important for horse racing, trainers, jockey and other personnel. The split timing system would provide fans with reference data, provide trainers with training intensity indicators, and provide jockey with walking strategy support in horse racing.

- It is suggested that all events in the country should be connected in an organic way. A national database platform should be set up to store and statistically analyze the split time of all the participating horses in all races anywhere. And then, it would provide technical services for the national races.

\section{REFERENCES}

[1] Wang Lingao. Study on CCD timing system of multi-segment high speed of track training [J]. Technology of Computer Application. 2010(3): 813-814.

[2] Xing Yu. The theory and practice of high-level sprint training based on precise split timing [J]. Journal of Chengdu Sport University. 2014(6): 51-56.

[3] Ji Guosheng. Design and implementation of manual split timing method of straight sprint [J]. Athletics. 2015(12): 47-49.

[4] Ma Mingda. Sports betting in Macau [J]. Sports Culture Guide [J]. 2006(4).

[5] Wang Hong, Zhong Haiou, Qiu Chengyao. Review and development trend of sports betting industry [J]. Journal of Guangzhou Sport University. 2000(3).
[6] Wu Yigang. The influences of the development of American sports betting on Chinese sports lottery [J]. Sports Culture Guide. 2003(11).

[7] Xu Na, Li Hai, Zong Zhiwei, el at. The social responsibility of Internet sports betting company [J]. Journal of Shenyang Physical University. 2011(4). 